\title{
A surprising method for polarising antiprotons
}

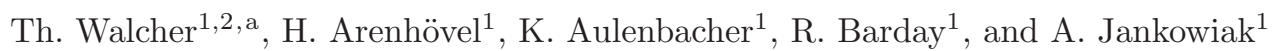

1 Institut für Kernphysik, Johannes Gutenberg-Universität Mainz, D-55099 Mainz, Germany

2 Laboratori Nazionali di Frascati, Istituto Nazionale di Fisica Nucleare, I-00044 Frascati (Rome), Italy

Original article: Eur. Phys. J. A 34, 447 (2007) DOI: 10.1140/epja/i2007-10462-x

Received: 12 December 2008

Published online: 5 January 2009 - C Società Italiana di Fisica / Springer-Verlag 2009

Triggered by a criticism of A.I. Milstein, S.G. Salnikov and V.M. Strakhovenko [1] we have searched for the reason of the dramatic difference of our triple-spin transfer cross-section in the scattering of spin- $1 / 2$ hadrons from leptons [2]. We found a numerical problem in our calculation of the spin-dependent cross-sections $[3,4]$. After the correction of this problem the cross-sections are smaller by about 14 orders of magnitude. The corrected figs. 1-4 of ref. [2] are repeated in this erratum (in the following page).

Though some features as the ratio $\left\langle S_{2}^{-}\right\rangle /\left\langle S_{2}\right\rangle=0.49$ are more favorable now, the huge reduction of the cross-section kills the proposal. In order to arrive at similar polarisation times and figures of merits as in ref. [2] an increase of the positron density of about 13 orders of magnitude had to be achieved. As a realistic increase four orders appear to be possible today [5].

However, there still remains a difference between our and the calculation of ref. [1]. Whereas we get $\sigma_{-\lambda, \lambda,+}^{h} \approx$ 30 mbarn for $\lambda=-$ and 0.5 mbarn for $\lambda=+$ at $T_{h}=1.7 \mathrm{keV}$ A.I. Milstein, S.G. Salnikov and V.M. Strakhovenko get $\sigma_{\text {total }}=\sigma_{-,+,+}^{h}+\sigma_{+,-,+}^{h}=0.75$ mbarn. This difference may be due to their averaging over all relative initial directions of the hadron momentum with respect to the lepton spin.

\section{References}

1. A.I. Milstein, S.G. Salnikov, V.M. Strakhovenko, Nucl. Instrum. Methods B 266, 3453 (2008) [arXiv:0802.3766 [hep-ph]].

2. Th. Walcher, H. Arenhövel, K. Aulenbacher, R. Barday, A. Jankowiak, Eur. Phys. J. A 34, 447 (2007) [arXiv:0706.3765 [physics.acc-ph]].

3. H. Arenhövel, Eur. Phys. J. A 34, 303 (2007) [arXiv:0706.3576 [nucl-th]].

4. H. Arenhövel, Erratum to Eur. Phys. J. A 34, 303 (2007) (this issue).

5. K. Aulenbacher, H. Arenhoevel, R. Barday, A. Jankowiak, T. Walcher, AIP Conf. Proc. 980, 170 (2008).

\footnotetext{
${ }^{a}$ e-mail: walcher@kph.uni-mainz.de
} 


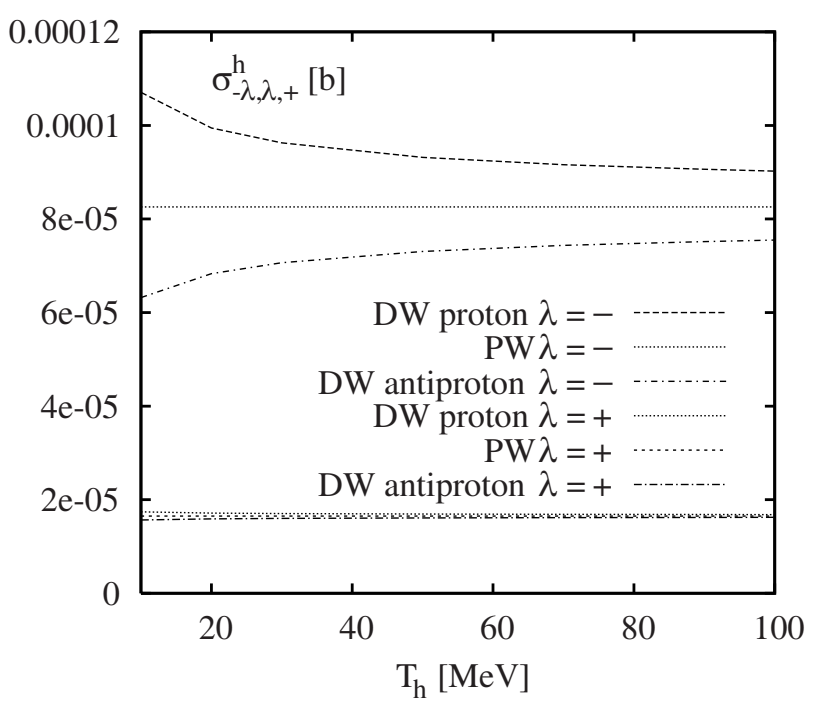

Fig. 1. The integrated spin-flip cross-sections $\left\langle\sigma_{-\lambda, \lambda,+}^{h}\right\rangle$ $(\lambda= \pm)$ for antiproton and proton electron scattering as a function of the proton lab kinetic energy $T_{h}$ for $b=10^{10} \mathrm{fm}$ in PW and DW. The cross-section for proton and antiproton are identical in PW. It is easy to calculate the lepton kinetic energy in the proton rest system using the nonrelativistic relation $T_{l}=T_{h}\left(m_{l} / m_{h}\right)$.
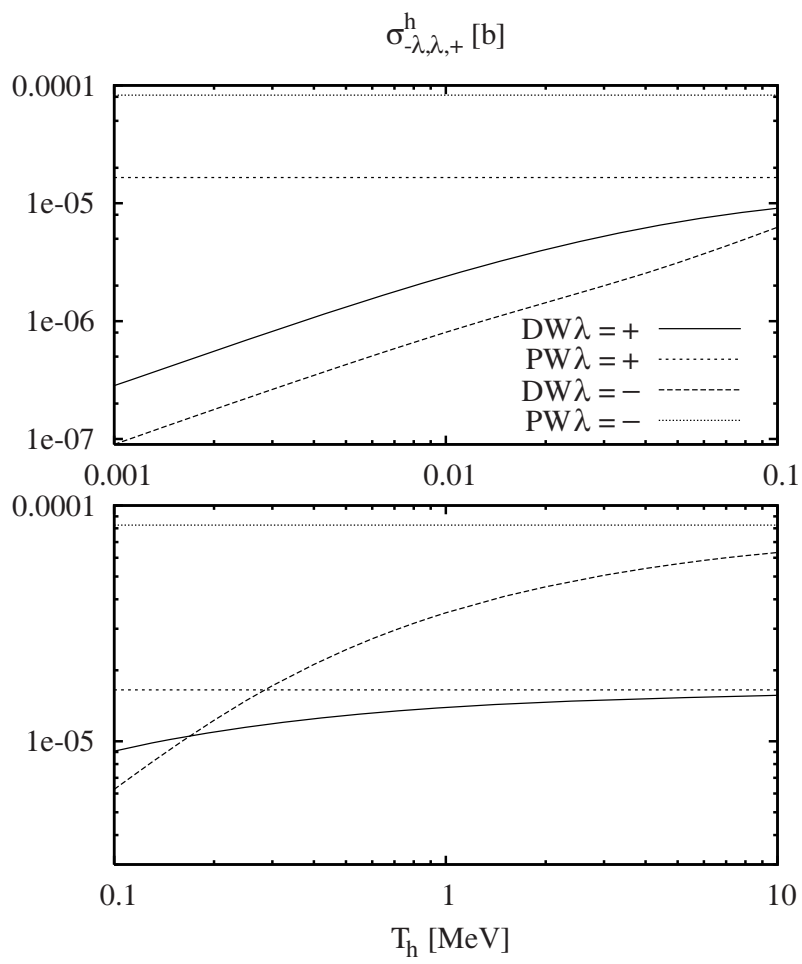

Fig. 2. The integrated spin-flip cross-sections $\left\langle\sigma_{-\lambda, \lambda,+}^{h}\right\rangle$ $(\lambda= \pm)$ for antiproton electron scattering as a function of the proton lab kinetic energy $T_{h}$ for $b=10^{10} \mathrm{fm}$ in PW and DW.

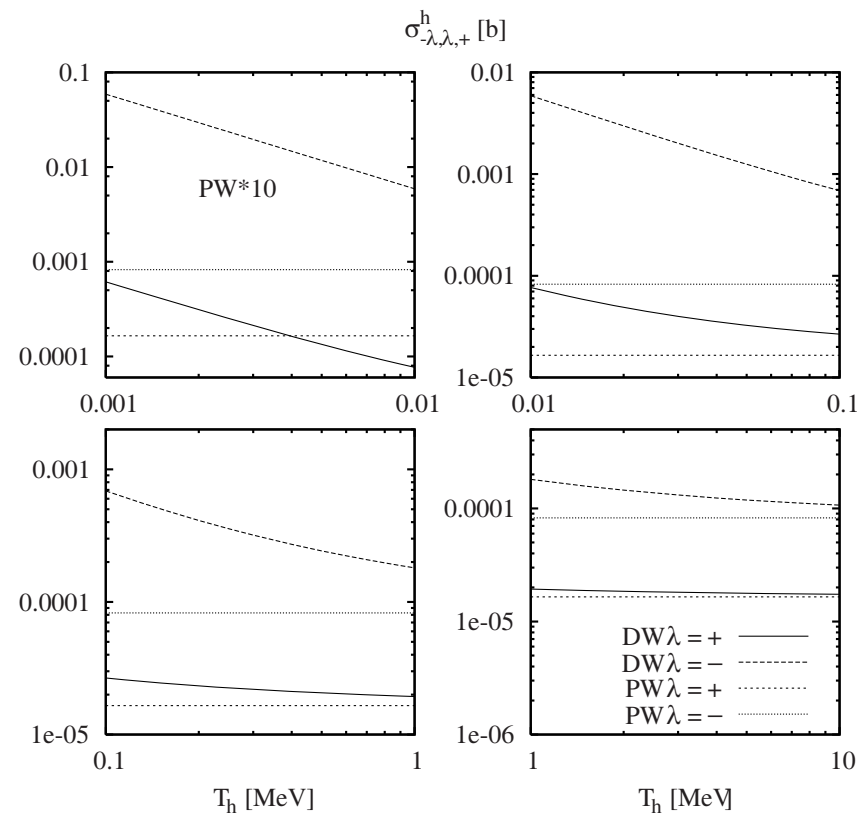

Fig. 3. The integrated spin-flip cross-sections $\left\langle\sigma_{-\lambda, \lambda,+}^{h}\right\rangle$ $(\lambda= \pm)$ for proton electron scattering as a function of the proton lab kinetic energy $T_{h}$ for $b=10^{10} \mathrm{fm}$ in PW and DW.

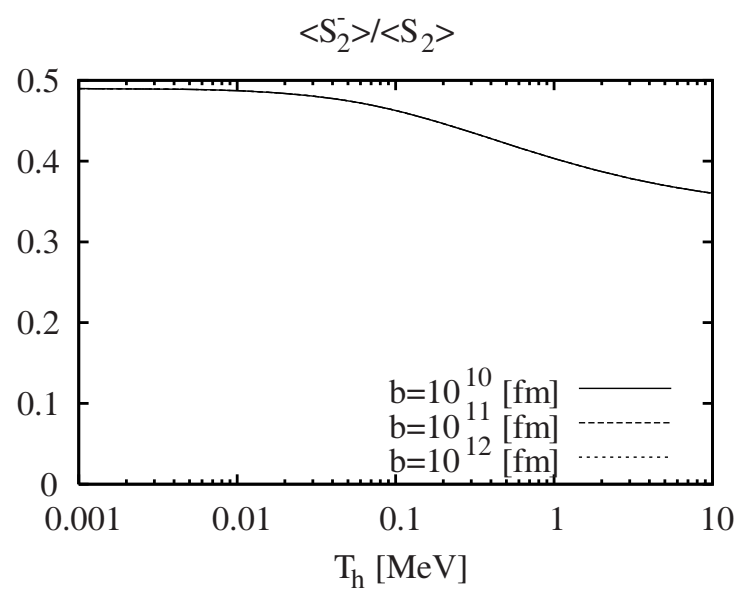

Fig. 4. Ratio of $\left\langle S_{2}^{-}\right\rangle /\left\langle S_{2}\right\rangle$ for proton electron scattering as a function of the proton lab kinetic energy $T_{h}$ for the DW approximation. 